\title{
Teaching postgraduates about managing drug and alcohol misuse
}

\author{
Report from UK medical royal colleges outlines the key competencies
}

\author{
Zoe Morris-Williams clinical lecturer ${ }^{1}$, Lynn Monrouxe senior lecturer ${ }^{1}$, Andrew Grant clinical senior \\ lecturer ${ }^{1}$, Adrian Edwards professor ${ }^{2}$
}

${ }^{1}$ Institute of Medical Education, School of Medicine, Cardiff University, Cardiff CF14 4YS, UK; ${ }^{2}$ Cochrane Institute for Primary Care and Public Health, School of Medicine, Cardiff University

A working group of the UK medical royal colleges reported recently on the core competencies needed by all postgraduate specialist trainees regarding substance misuse. ${ }^{1}$ This follows other recent reports, including those from individual royal colleges, parliament, and other stakeholders, that highlight the need to change the system by which we recognise and manage drug and alcohol misuse. Such changes were implemented in the undergraduate curriculum after the International Centre for Drug Policy produced guidance on teaching about substance misuse in 2007. ${ }^{2}$ However, the knowledge and skills learnt at medical school are not yet part of the core competencies of postgraduate medical training in the United Kingdom.

The increasing use of illicit drugs and the hazardous consumption of alcohol provide a growing challenge for the NHS. In 2009-10, more than one million hospital admissions in England were alcohol related, ${ }^{3}$ at a cost of almost $£ 3 b n$ (€3.8bn; $\$ 4.7 \mathrm{bn}) .{ }^{4} \mathrm{It}$ is estimated that in England alone, $25 \%$ of the adult population (7.6 million people) drink hazardous amounts of alcohol and almost half of them show signs of alcohol related ill health. ${ }^{4}$ In addition, around $9 \%$ of adults questioned as part of the British crime survey 2010-11 admitted to illicit drug use at least once in the past year. ${ }^{5}$ As numbers of cases increase so too do costs and workloads.

Of specific concern, clinicians often miss opportunities to identify and provide effective interventions for people with harmful drinking and drug use. Only about $6 \%$ of people with alcohol dependency in England receive appropriate treatment, perhaps because clinicians fail to ask patients about their drug and alcohol use in routine consultations. ${ }^{6}$

The royal colleges working group's core competencies for all postgraduate trainees have been grouped into the three domains common to most postgraduate curriculums - knowledge, skills, and attitudes and behaviour (summarised in the box). ${ }^{1}$

As with any new intervention in medical training, setting out the core competencies is just the starting point of a longer and more complex journey. The way in which the competencies are taken up and integrated into the existing curriculums, teaching programmes, and assessment programmes of the specialty training schools will be key to the success of the endeavour. The report's aim of introducing these competencies across all specialty schools highlights not only the importance of the problem of substance misuse, but also that it is a problem within all healthcare disciplines, from child health and paediatrics through to the care of older adults. The report also serves as a reminder to all royal colleges that attitudes to dealing with drug and alcohol misuse need to change throughout the medical profession. Improving the detection and treatment of this increasingly common condition is the responsibility of all doctors, and any potential conflicts between personal attitudes and professional duties must be tackled. These competencies would not be complete if they did not cover the prevalence of alcohol and drug misuse among doctors, sources of help for doctors with alcohol and drug problems, and the obligation to act if a colleague's drinking or drug use threatens patient care. The report is rightly ambitious in deciding that competencies should be implemented across all specialties, each with their very different curriculums, training requirements, assessment tools, and teaching arrangements. Although all the royal colleges have adopted modern educational approaches that include supervised training and e-portfolio resources, methods of teaching and the emphasis given to face to face teaching vary. For example, some specialty trainees attend weekly teaching sessions whereas others attend less frequent, day long regional training days. The report does not mention how the core competencies will be delivered or assessed. A multifaceted approach will be needed-probably a combination of face to face teaching, simulated patients, and instruction on conducting motivational interviewing and "brief intervention" techniques. ${ }^{78}$ In addition, it will be important for doctors to know that a multidisciplinary approach (psychology, clinical pharmacology, and specialist nurse input) should be used. A robust system of assessment that will drive the desired change in behaviour (implementation of the competencies and change in practice) 


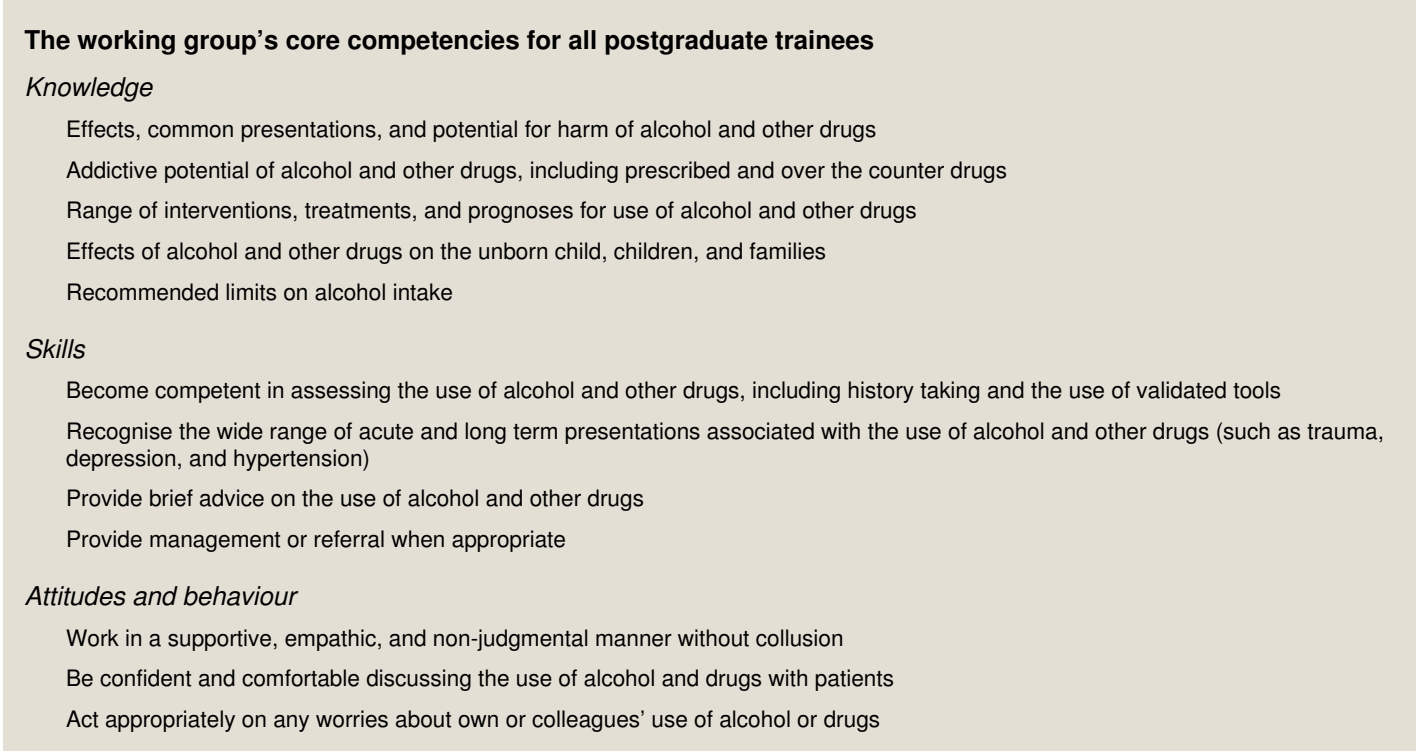

rather than just confirm that the information has been delivered will be necessary. The report does not mention how such extra multifaceted teaching and assessment should be financed. Currently, the delivery and teaching of specialty training curriculums is largely organised at the level of the deanery or hospital trust and large variations exist. Inconsistency could undermine the quality of the training for these complex skills, however.

The royal colleges should be praised for uniting in their efforts to make prevention, screening, and early intervention for the misuse of drugs and alcohol the responsibility of every doctor, regardless of the route they take to gain specialty registration. ${ }^{9}$ However, some key areas for discussion remain to ensure that their laudable aims do not fall victim to the tick box culture that is prevalent in postgraduate medical training.

Competing interests: All authors have completed the ICMJE uniform disclosure form at www.icmje.org/coi_disclosure.pdf (available on request from the corresponding author) and declare: no support from any organisation for the submitted work; no financial relationships with any organisations that might have an interest in the submitted work in the previous three years; no other relationships or activities that could appear to have influenced the submitted work.
Provenance and peer review: Commissioned; not externally peer reviewed.

1 Royal College of Psychiatrists. Alcohol and other drugs: core medical competencies. Final report of the Working Group of the Royal Colleges. 2012. www.rcpsych.ac.uk/publications collegereports/op/op85.aspx.

2 International Centre for Drug Policy. Substance misuse in the undergraduate medical curriculum . 2007. www.sgul.ac.uk/research/projects/icdp/our-work-programmes/pdfs/ substance-misuse-book.pdf.

3 NHS Information Centre. Statistics on alcohol: England, 2011. Health and Social Care Information Centre. 2011. www.ic.nhs.uk/webfiles/publications/003_Health_Lifestyles/ Alcohol_2011/NHSIC_Statistics_on_Alcohol_England_2011.pdf.

4 National Audit Office. Reducing alcohol harm: health services in England for alcohol misuse. 2008. www.nao.org.uk/publications/0708/reducing_alcohol_harm.aspx.

5 Smith K, Flatley J, eds. Drug misuse declared: findings from the 2010/11 British crime survey. England and Wales (Home Office statistical bulletin 12/11). 2011. www.homeoffice. gov.uk/publications/science-research-statistics/research-statistics/crime-research/ hosb1211/hosb1211?view=Binary.

6 Home Office. Drug strategy 2010: reducing demand, restricting supply, building recovery. Supporting people to live a drug-free life. 2010. www.homeoffice.gov.uk/publications/ alcohol-drugs/drugs/drug-strategy/.

7 Miller RW, Rollnick S. Motivational interviewing: preparing people to change addictive behaviour. Guilford Press, 1991.

8 Kaner EF, Beyer F, Dickinson HO, Pienaar E, Campbell F, Schlesinger C, et al. Effectiveness of brief alcohol interventions in primary care populations. Cochrane Database Syst Rev 2007;2:CD004148.

9 National Institute for Health and Clinical Excellence. Alcohol-use disorders-preventing the development of hazardous and harmful drinking (PH24). 2010. http://guidance.nice. org.uk/PH24. 